\title{
Notas sobre los partidos políticos en el Brasil contemporáneo
}

Víctor Manuel Durand Ponte IIS-UNAM

En este artículo se analiza cómo la debilidad del sistema político brasileño ha constituido un criterio constante según la revisión que el autor hace de diferentes textos históricos.

Sin embargo la formación de identidades partidarias más fuertes y permanentes permite vislumbrar la afirmación de los partidos políticos y una consolidación de la democracia.

$\mathrm{L}$ os partidos políticos, como instituciones que vinculan a la ciudadanía con el Estado, encuentran su importancia dentro de los regímenes democráticos; fuera de ellos tienen una escasa relevancia, como bien lo muestra el trabajo de Waldo Ansaldi, o cumplen otras funciones como, por ejemplo, durante las dictaduras o el periodo oligárquico, en los cuales los partidos suelen presentarse como instrumentos de dominación de las clases dominantes y no como mecanismos de representación. Si aceptamos la definición de la democracia moderna como un método para la se- lección de los gobernantes por los ciudadanos, ${ }^{1}$ los partidos políticos resultan, pese a su crisis, una institución fundamental tanto para que las elites puedan presentar sus opciones políticas, ideológicas y programáticas a la ciudadanía, como para que los propios ciudadanos puedan realizar su elección entre las distintas ofertas. El conjunto de partidos se agrupan necesariamente en sistemas específicos ${ }^{2}$ que definen las reglas de la competencia política, los derechos y obligacio-

1 Shumpeter, Capitalismo, 1961, p. 61.

2 Sartori, Partidos, 1980 , p. 80. 
nes de los militantes y representantes dentro de ellos, el número de organismos partidarios que conforman el sistema, etcétera. Dichos sistemas varían mucho de país a país sin que se pueda hablar de un cierto modelo básico o típico. Más bien lo que se espera de un sistema de partidos es que cumpla sus funciones y garantice la estabilidad del régimen a largo plazo.

El tema de los partidos políticos encuentra una especial relevancia en los procesos de transición política en los cuales las instituciones políticas o no existen o son insuficientes o están profundamente impregnadas de sus antecedentes autoritarios. La construcción de instituciones propiamente democráticas, la ingeniería política como se le ha llamado, se vuelve un requisito para que el régimen democrático encuentre su estabilidad y consolidación, amén, por supuesto, de otros requisitos como es la existencia de una cultura democrática entre los públicos de masas.

En Brasil encontramos un caso en donde dicho sistema ha sido calificado permanentemente de crítico o insuficiente. Esta afirmación es válida no sólo para el periodo predemocrático estudiado en el trabajo de Waldo Ansaldi, sino hasta el presente, aun en la última elección presidencial de 1994, en que se acusó a los partidos y al sistema de partidos de ser débiles y desempeñar mal sus funciones. ${ }^{3} \mathrm{Co}$ mo afirma Scott Mainwaring, ${ }^{4}$ el caso de Brasil es un caso extremo de subdesarrollo partidario. Quizá una forma

${ }^{3}$ Meneguello, "Partidos", 1994, pp. 151 ss.

4 Mainwaring, "Partidos", 1989, p. 69. extrema de señalar dicha crisis sea la afirmación de Huntington en el sentido de que Brasil, hasta 1979, como otros países de América Latina, no alternan sistemas democráticos y autoritarios, sino que la propia alternancia constituye el sistema político, dentro del cual los partidos obviamente serían secundarios. ${ }^{5}$

Las explicaciones que se han aducido en la literatura especializada son de distinta índole. Para algunos el subdesarrollo de los partidos políticos es permanente en la historia de Brasil, entre 1889 y 1945 prácticamente no existió ninguna competencia entre los partidos existentes, la competencia se daba más entre personas y entre familias. Hasta el final del Estado Nuevo en 1945 , los líderes estatales y las oligarquías regionales actuaban deliberadamente en contra de la construcción de partidos políticos nacionales que ponía en peligro su control político local. ${ }^{6}$ En el caso de Vargas, su oposición a los partidos políticos sólo cedió en 1943 cuando, a causa de la posguerra, el apoyo partidario se volvió indispensable para mantenerse en el poder. ${ }^{7}$

Si la oposición a los partidos políticos por parte de los oligarcas o el dictador Vargas es fácilmente comprensible por ser regímenes que excluían la participación ciudadana y sus respectivas mediaciones, también lo es el hecho de que durante el periodo que va de 1945 a 1964 , es decir durante el primer periodo democríti-

\footnotetext{
SIIuntington, Terceira, 199/, p. 50.

"Ansaldi, "Caso", 1995.

7 Souza, Estallo, 1976.
} 
co, los partidos políticos hayan continuando siendo instituciones secundarias dentro del sistema político. Para María do Carmo Campello de Souza, el bajo desempeño de los partidos se debía a la existencia de una estructura de Estado centralizado antes del surgimiento del sistema de partidos, por lo cual su institucionalización se volvió muy dificil, y en contrapartida estimuló el fortalecimiento de los sistemas clientelares. A pesar de que Vargas fue retirado del poder en 1945, las estructuras corporativas del Estado Nuevo permanecieron intactas, lo que reforzó la centralización y estrechó el espacio de los partidos.

Una explicación más compleja la encontramos en el trabajo de Lamonier y Meneguello; ${ }^{8}$ para estos autores existen tres factores que operaron en contra del sistema de partidos en el periodo posterior a 1946. En primer lugar está el argumento señalado por Souza en el sentido de la presencia de un Estado centralizado que domina a los partidos. En segundo lugar el excesivo clientelismo que impidió la articulación de intereses de grupos o clases, debilitando el carácter representativo de los partidos. En tercero y último lugar señalan que, debido al menos en parte al sistema electoral, los políticos buscaban mantener su autonomía y su desarrollo individual antes que fortalecer a los partidos políticos. A todo ello habría que añadir la formación desde principios de siglo de una cultura política autoritaria, antiliberal y antipluralista?

8 Iamonicr y Mencguello, Partidos, 1986.

9 Moisés, "Democratizaçĩo", 1994.
Al lado de las explicaciones históricas y que abarcan el periodo completo de la vida independiente, tenemos las explicaciones particulares a cada sistema de partidos, correspondientes a las distintas épocas. Para el periodo de 1945 a 1964, Santos ${ }^{10}$ indica, como un factor importante para comprender la debilidad de los partidos, el fraccionamiento progresivo que sufrieron, especialmente el PSD, lo que llevó a una dispersión de los factores del poder y al correspondiente debilitamiento de la fuerza electoral de los partidos. De la misma manera existe un consenso entre los autores acerca de que el pluralismo polarizado que define al sistema de partidos inviabilizó la continuidad del régimen democrático. En efecto, la oposición entre populismo y antipopulismo reflejaba claramente la presencia del Estado en el control de los partidos populistas, Partido Social Democrático (PSD) y Partido Laborista Brasileño (P'lB), creados por Vargas desde las alturas del estado y la Unión Democrática Nacional (UDN) que fue creada por los sectores liberales para oponerse al populismo.

Junto a estas argumentaciones de carácter político hay otras que aducen criterios estructurales, como el de que los partidos políticos no pudieron soportar los efectos de la crisis económica que asoló al Brasil en los años sesenta. ${ }^{11} \mathrm{O}$ aquellas otras en que explican el derrumbe de la democracia por la existencia de una conspiración

10 Santos, Sessenta, 1987.

11 O'Donell, Sessenta, 1973 y Cardoso, "Associatcd", 1973. 


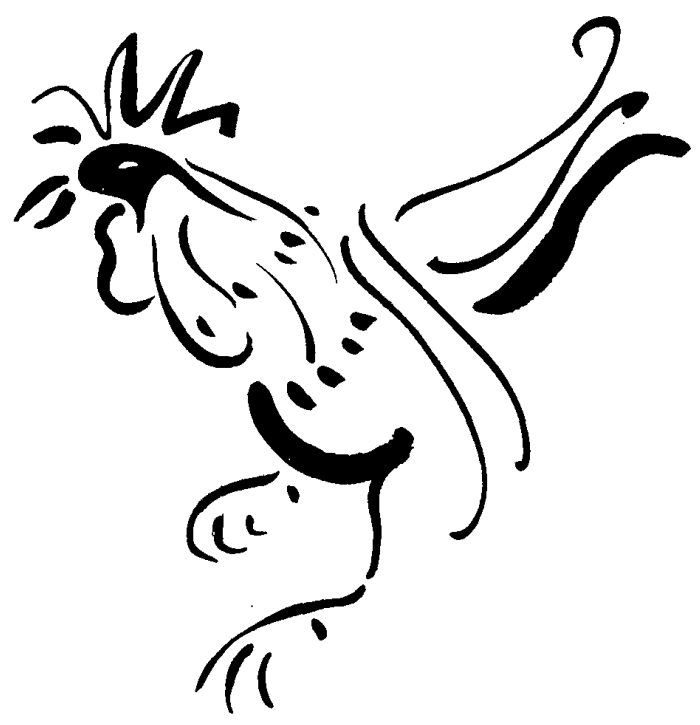

internacional y de la derecha en contra del gobierno de Goulart y que gracias a sus alianzas y eficacia logró su objetivo. Finalmente cabe señalar la argumentación de Stepan ${ }^{12}$ sobre la actuación del presidente Goulart y cómo el régimen fue siendo sometido a presiones macropolíticas que acabaton derrumbándolo. Estas últimas interpretaciones de carácter estructural o intencional excluyen prácticamente a los partidos políticos como actores relevantes del proceso de caída del régimen democrático, lo cual parece exagerado como lo ha mostrado Argelina C. Figuereido ${ }^{13}$ en su importante trabajo sobre la crisis del régimen de-

12 Stepan, Military, 1971.

13 Figuercido, Democracia, 1993. mocrático, en el cual muestra la inexistencia de determinismos y cómo sí hubo diferentes alternativas de acción que pudieron producir desenlaces diferentes.

En todo caso hay un consenso acerca del subdesarrollo del sistema de partidos en el Brasil del primer perio. do democrático.

Ya dentro de la dictadura militar de 1964 los partidos políticos fueron tolerados durante el primer año, no obstante las inesperadas derrotas en las elecciones de 1965 donde la oposición ganó en los estados de Guanabara y Minas Gerais. Los triunfos fueron atribuidos más a la fuerza de ciertos políticos como Juscelino Kubistchek y Carlos Lacerda, que a la capacidad de los partidos; no obstante, dicha 
experiencia electoral bastó para que el gobierno militar suprimiera el antiguo sistema de partidos de pluralismo polarizado e impusiera un bipartidismo tutelado que por definición sería también polarizado. La oposición entre populismo y antipopulismo cedió su lugar a la oposición entre autoritarismo o defensa del statu quo representado por la Alianza Renovadora Nacional (ARENA) y el antiautoritarismo defendido por el Movimiento Democrático Brasileño (MDB). En la ARENA se aglutinaron los legisladores pertenecientes a la antigua UDN y la fracción conservadora del PSD, mientras que los del PTB y los radicales del PSD lo hicieron en el MDB.

Es importante señalar, de acuerdo con Maria D'Alva Gil Kinzo, ${ }^{14}$ que la permanencia del MDB se debió en buena parte a la iniciativa de los militares que lo apoyaron de varias maneras, desde el hecho de entusiasmar a algunos diputados y senadores a incorporarse al partido de oposición, hasta el de abrirle ciertos espacios de oposición moderada. El carácter polarizado del sistema de partidos obligó al MDB a asumir lentamente posiciones de un verdadero partido de oposición, lo cual desde luego fue una-tarea muy dificil. Al inicio de la dictadura, y hasta 1977 , la acción del partido estuvo marcada por la necesidad de evitar que la represión de los militares cayese sobre los partidos o sus miembros $y$, al mismo tiempo, el mantener un discurso muy moderado era castigado por la ciudadanía, como se mostró claramente en las elecciones de 1970

14 Kinzo, “Opposition”, 1985. cuando el MDB sufrió una devastadora derrota.

Pese a todas las dificultades y las prerrogativas que la ley otorgaba a la AREN $\Lambda$, el MDB fue conformándose como un frente antiautoritario que participaba en elecciones claramente plebiscitarias, que calificaban al régimen autoritario. Así, pese a su debilidad como partido, el MDB fue influenciando al gobierno militar y se convirtió en una pieza fundamental de la transición a la democracia.

El crecimiento del MDB como receptáculo de las fuerzas antiautoritarias fue incrementándose a partir de 1974 hasta 1978 , cuando sus triunfos se extienden a todas las grandes ciudades del Brasil. En 1979 el gobierno militar realizó una nueva reforma al sistema de partidos con la finalidad de romper la lógica plebiscitaria que habían adquirido las elecciones y creó un sistema de partidos de pluralismo limitado. La ARENA se convierte en Partido Democrático Social (PDS), el MDI logra mantener la sigla en el nuevo sistema al denominarse PMDB (Partido del Movimiento Democrático Brasileño), creándose además el Partido de los Trabajadores (PT), el PTB y el Partido Democrático Laborista (PDL), el Partido Popular (PP) y el Partido Socialista Brasileño (PSI). Sin embargo, y pese a la reforma partidaria de 1979, el gobierno no logró destruir el frente antiautoritario comandado por el PMDB, que en 1985, con el apoyo de otros partidos, logró derrotar al PDS en la elección del nuevo presidente de la república.

Durante el gobierno militar continuaron operando algunos de los fac- 
tores que ya vimos que explican el subdesarrollo de los partidos políticos. El centralismo del Estado y su dominio sobre la vida partidaria es más importante que en el pasado y, pese a la evolución que adoptan las elecciones, los gobiernos militares no abandonan nunca su control y manipulación sobre los partidos y su sistema. Así mismo, como contraparte de la centralización, se reprodujo el clientelismo de los legisladores como forma de vinculación con la población, dando lugar incluso al surgimiento de la llamada política fisiológica, que sanciona justamente dichas prácticas clientelares y el control de las máquinas partidarias mediante el control de poblaciones cautivas.

Durante el periodo del pluralismo limitado se creó el Partido de los Trabajadores $\left(\mathrm{P}^{\top} \mathrm{T}\right)$, que es una organización clasista, con una línea política de oposición intransigente a los diferentes gobiernos del periodo militar y a los tres siguientes (José Sarney, Fernando Collor e Itamar Franco), y con un programa basado en argumentos de carácter político social y éticos, con lo cual define una nueva forma de organización partidaria con un control importante sobre sus miembros, con una preponderancia de los individuos sobre los militantes y los políticos electos por la sigla. Desde entonces hasta la fecha, el PT se ha conformado como el único partido fuerte orgánicamente y con un electorado racionalmente orientado a sus programas.

A partir de 1985 se modificó la legislación, y del sistema de pluralismo limitado se pasó a uno de pluralis- mo permisivo, como acertadamente lo ha denominado Álvaro Moisés. ${ }^{15}$ No obstante, el multipartidarismo no puso fin a la polarización en la competencia partidaria debido al establecimiento de dos turnos para definir las elecciones, lo cual hace que en la segunda vuelta se enfrenten necesariamente coaliciones de partidos radicalmente opuestos.

La estructura partidaria sufrió una drástica alteración con la facilidad con que se podían construir nuevas agrupaciones partidarias. El PMDB se dividió por razones programáticas, dando lugar en 1987 al Partido Socialdemócrata Brasileño (PDSB), actualmente en el poder. El PDS también se fraccionó, dando lugar al Partido del Frente Liberal (PFL), y más tarde el propio PISS se transformó en el PPR. Junto al fraccionamiento de los antiguos partidos se legalizaron viejos partidos que permanecían en la ilegalidad como el Partido Comunista Brasileño (PCB) o el Partido Comunista do Brasil (PCdoß), y se crearon nuevos como el Partido de Reedificación del Orden Nacional (PRONA), el Partido de Renovación Nacional (PRN) de Collor, y muchos otros que sería muy largo enumerar.

En el periodo de la Nueva República, que se inaugura con la elección indirecta del presidente de la república en 1985 siendo electo Tancredo Neves, los partidos políticos pasan a sufrir nuevas exigencias tanto en el mundo de la política como en las necesidades de representación de la sociedad. En el gobierno de Sarney, quien asumió la presidencia después

15 Moisés, op. cit. 
de la muerte de Tancredo Neves, los partidos políticos se enfrentaron al problema de crear la nueva Constitución Política del Brasil, proceso en el cual la definición del Congreso en términos de filiación partidaria se desdibujó, lo que resultó dramático en el caso del PMDB, y dio lugar a reagrupaciones y arreglos definidos en torno a la política del presidente y de los gobernadores; en este proceso destacó la creación del llamado "centrão", que aglutinó a legisladores de varios partidos contrariando las directrices de los líderes. Los partidos políticos fueron incapaces de presentar frentes articulados ante las presiones de la presidencia. La propia Constitución reflejó la presión de los grupos parlamentarios de interés que introdujeron una cantidad de normas quie responden más a intereses sectoriales o particulares que generales.

En el plano electoral los cambios también fueron mayores, y la exigencia sobre los partidos creció significativamente. Por una parte el fin de la dictadura dio término a la oposición entre autoritarios y antiautoritarios, redefiniendo la percepción de los ciudadanos de los partidos políticos. Sin embargo, la selección de los candidatos por parte de la población no respondía a criterios programáticos o partidarios, sino que continuó siendo con base en la evaluación, fundamentalmente económica, de los gobiernos $y$, por consiguiente, de los partidos de la situación. Este hecho sacrificó al PMDB, que en la elección de 1988 obtuvo menos del $10 \%$ de los votos, cuando en 1985 había ganado en la gran mayoría de las elecciones para elegir gobernadores. El voto de castigo conformó una nueva pauta de comportamiento político generalizado, con lo cual apareció en el escenario político un nuevo fenómeno: la volatilidad del electorado o la conformación de mayorías volátiles, que se manifestaron con claridad en las elecciones de 1988 cuando fue electo Fernando Collor, y en las de 1994, cuando ganó la presidencia Fernando H. Cardoso.

En el caso de Fernando Collor, la negación de los partidos como vehículos de representación fue innegable; el candidato se eligió presidente sustentado por un nuevo partido creado ad hoc, el Partido de Renovación Nacional (PRN), y con un discurso francamente antipolítico y antipartidario; la caza de los "marajas", de los corruptos, simbolizó esa negación. ${ }^{10}$ La elección de Fernando H. Cardoso no tiene ese matiz antipartidario; por el contrario, el candidato valorizó la política, pero el apoyo que recibió fue, en buena medida, producto del éxito del Plan Real que abatió la inflación, que fue producto de un plan o programa, de gobierno. ${ }^{17}$ El propio Fernando $\mathrm{H}$. Cardoso ha dicho que la política funcionó como un mito donde existen el bueno (el Plan) y el malo (la inflación), y que ese mito fue el que permitió establecer el vínculo con la población.

A pesar de la persistencia de la debilidad del sistema de partidos aún presente, cabe destacar que en el pe-

16 Sallum, Gracif y Gomes, "Blcições", 1990, pp. 69-88.

17 Mencgucllo, op. cit. 
riodo de la Nueva República se han conformado identidades partidarias más fuertes y permanentes, el caso ejemplar es el del PT, donde la cultura política se está orientando de manera más positiva hacia los partidos políticos. Sin embargo, está presente la necesidad de un cambio en la legislación acerca de los partidos políticos que favorezca más su desarrollo y posibilite la consolidación de la democracia, poniendo fin a las mayorías volátiles y generando identidades políticas más definidas y duraderas.

\section{BibLIOGRAFIA}

- Ansaldi, Waldo, "Un caso de ficción de organización partidaria o la política sin partidos: Brasil, 1889-1945", en este volumen, 1995.

-Cardoso, Fernando H., "Associateddependence development: Theoretical and practical implications", en Alfred Stepan (comp.), Autorilarian Brazil, Yale University Press, New Haven, 1973.

- Figucreido Cheibub, Argelina, Democracia ou reformas? Alternativas democrálicas a crisis política: 1961-1964, Paz. c Terra, São Paulo, 1993.

-Huntington, Samuel, A Terceira Onda, a democratizacão no final do seculo $X X$, Atica, São Paulo, 1994.

-Kinzo, Maria D'Alba Gil, "An opposition party in an autorithariam regime: The case of the MDB in Brazil, 1966-1979", tesis de doctorado, Oxford University, 1985.

-Lamonier, Bolivar, y Rachel Maneguello, Partidos políticos e consolidão demo- cratica: O caso de Brasil, Ed. Brasilense, São Paulo, 1986.

-Mainwaring, Scott, "Partidos políticos e democratizacão no Brasil e no Cono Sul", en Aida De Souza, Democracia, partidos e cultura política na América Latina, Editora Kuarup, Porto Alegre, 1989.

-Menegucllo, Rachel, "Partidos c tendencias de comportamento: o cenario político en 1994", en Evelina Dagnino (coord.), Anos 90 politica e sociedade no Brasil, Brasiliense, São Paulo, 1994, pp. 151 ss.

-Moisés, José Álvaro, "Democratização c partidos políticos no Brasil", mimco, Departamento de Ciencial Política, USP, São Paulo, 1994.

-O'Donnell, Guillermo, Sessenta e Quatro: Anatomia da crise, Rio de Janeiro. Mothemization na Burocracy-Autorilarianism, Institute of International Studens, Berkelcy, 1973.

-Sallum Jr., Brasilio, Eduardo Graelf; Elisabeth de Lima Gomes, "Eleições presidenciais e crise do sistema partidario", Lua Nova, CEDEC, núm. 20, Sao Patulo, 1990 , pp. 69 a 88.

-Santos, Wanderley Guilherme dos, Sessenta e Quatro: Anatomia da Crise, Río de Janeiro, 1987.

-Sattori, Giovanni, Partidos y sistemas de partidos. Marco para un análisis, 2 volúmenes, Alianza Editorial, Madrid, 1980.

-Shumpeter, Joseph A., Capilalismo, socialismo e democracia, londo de Cultura, Río de Janeiro, 1961.

-Souza, Maria do Carmo Campello de, Estado e partidos politicos no Brasil (1930 a 1964), Alfa-Omega, São Paulo, 1976.

-Stepan, Alfred, The military in polttics: changing paterns in Brazil, Princcnton University Press, Princeton, 1971. 